\title{
Fractured Reservoir Simulation: a Challenging and Rewarding Issue
}

\author{
B. Bourbiaux \\ Institut français du pétrole, IFP, 1-4 avenue de Bois-Préau, 92852 Rueil-Malmaison Cedex - France \\ e-mail: bernard.bourbiaux@ifp.fr
}

\begin{abstract}
Résumé - Simulation des réservoirs fracturés : un défi et un enjeu - Au cours des années récentes, la prise de conscience du rôle des fractures sur la production et la récupération des champs est devenue de plus en plus forte au sein de la communauté pétrolière. Aussi beaucoup d'efforts ont-ils été consacrés à la détection des fractures et à l'analyse de leur impact sur la production. Toutefois, la prise en considération de ces observations dans les choix de développement des champs passe par la simulation de réservoir. Cet article traite des spécificités propres aux réservoirs fracturés et qui font de leur simulation à la fois un défi et un enjeu. En effet, l'intégration des fractures dans un modèle de simulation des écoulements n'est pas immédiate en raison du difficile passage à opérer entre l'observation géologique du réseau de fractures/failles et le rôle exercé par ce réseau sur des mécanismes de récupération souvent complexes. Sachant de plus que les fractures peuvent aussi bien freiner que promouvoir la production, la simulation des réservoirs fracturés peut être considérée comme un défi technique de grand enjeu. Cet article décrit ce contexte propre aux réservoirs fracturés en tant qu'introduction à deux articles techniques dédiés à la simulation de réservoir en double milieu. Bien qu'elle constitue un autre aspect majeur de l'étude de tout réservoir fracturé, la caractérisation géologique des fractures n'est pas discutée ici, mais seulement évoquée en raison d'une intégration croissante des aspects statique et dynamique.
\end{abstract}

\footnotetext{
Abstract - Fractured Reservoir Simulation: a Challenging and Rewarding Issue - The recent years have seen a growing awareness of the role played by fractures in petroleum reservoirs production and recovery. Hence, much effort was devoted to the diagnosis of fracture presence and impact on production. However, turning that diagnosis into field development decisions goes through reservoir simulation. This paper addresses some of the specificities of fractured reservoirs that make that their simulation is both challenging and rewarding. Indeed, the integration of fractures into a flow simulation model is not straightforward because of the existing gap between the geological fault/fracture network and the fingerprint of that network on often-complex recovery mechanisms. Considering that fractures may impede or enhance production, fractured reservoir simulation may be seen as a technical challenge with potentially-high reward. This paper underlines that specific framework as an introduction to two technical articles dedicated to dual-porosity reservoir simulation. Although it constitutes another major aspect of any fractured reservoir study, the geological characterization of fractures is not discussed herein, but only evoked because of more and more integration of static and dynamic aspects.
} 


\section{INTRODUCTION TO FRACTURED RESERVOIRS: SPECIFIC FEATURES - EXAMPLES - ECONOMIC CONTRIBUTION}

The occurrence of fractured reservoirs over the world is well acknowledged. A quite significant proportion of world oil reserves is commonly assumed to lie in fractured reservoirs, for instance Firoozabadi (2000) gives an estimation of more than over $20 \%$. Although not or less reported, the proportion is probably equivalent or higher for fractured gas reservoirs if one takes into account their higher depth in average, often involving a higher occurrence of diagenesis and fracturing phenomena.

Figures may fluctuate from one author to another because the classification of a reservoir as "fractured reservoir" is not straightforward as it depends on the classification criteria and also on the availability of reservoir information related to the presence of natural fractures. Regarding field exploitation, the determining criterion to consider a reservoir as fractured is not so much the presence of fractures but rather the impact of those fractures on the flow behaviour of the reservoir subjected to fluid extraction. Indeed, as Nelson (1985) clearly warns the reader in his book ("Geologic Analysis of Naturally Fractured Reservoirs"), "finding fractures is not enough" because a fractured reservoir is before all "a reservoir in which naturally occurring fractures either have, or are predicted to have, a significant effect on reservoir fluid flow either in the form of increased reservoir permeability and/or porosity or increased permeability anisotropy".

But, by the way, what are the specific features that define a fractured reservoir?

\section{Specific Features}

Regarding geology, fractures and faults can be defined as surfaces of discontinuity within a rock volume initiated under given stress conditions. They can act as conductive flowpaths or on the opposite as barriers, depending on the history of stress and fluid circulation following their genesis. They are present at all scales from the $100-\mathrm{km}$ basin scale down to the centimetre core scale. The existence of fault/fracture network at different scales was soon identified and quantified from fractal, i.e. self-similar scale-dependent models.

Depending on their throw and their intrinsic flow properties, faults may then be responsible for creating different compartments within the reservoir, hence their detection and characterization are essential for field delineation. Beyond that field appraisal stage, the reservoir engineer is concerned with the fractures or faults crossing a given hydrocarbon reservoir, defined as a fluid-bearing rock volume constituting a unique hydraulic entity.

The present paper addresses that reservoir engineering framework. Within that framework, a common classification of fractured reservoirs consists in considering the additional contribution of the matrix medium to the overall reservoir porosity and permeability. That classification implicitly assumes a role of fractures on the reservoir permeability, i.e. its productivity. Three main types of fractured reservoirs can then be defined:

- type (a) where the matrix is tight, with fractures ensuring both the reservoir porosity and permeability;

- type (b) where the matrix represents most of the reservoir porosity but does not contribute significantly to the overall reservoir permeability and productivity;

- type (c) where the matrix is porous and also contributes to the reservoir permeability with the fractures.

Although flow criteria drive reservoir engineers' definition of fractured reservoirs, it remains indispensable to keep in mind some major geological factors driving their occurrence, namely:

- at first, the tectonic setting: obviously, reservoirs located in structurally-complex provinces are more likely to have been subjected to intense deformation with faulting, folding and diffuse fracturing at a lower scale;

- the lithology: fractured reservoirs can be found in various sedimentary settings, however, carbonate formations are in average more often fractured than sandstone ones, for various reasons including different rock mechanical properties but also different susceptibilities to post-deposition evolution of the sediment;

- the reservoir age and its burial history, since older and/or deeply-buried formations are generally less porous and tighter, hence more prone to fracturing.

\section{Fractured Reservoirs Examples}

The following well-known fractured oil reservoir examples illustrate typical geological settings of fractured reservoirs:

- the Asmari porous limestone formation (OligoceneMiocene) in the foothills of Zagros mountain in Iran and North Iraq holds the most typical fractured carbonate reservoirs of the Middle East, generally of type (b), such as Haft Kel field with its nearly-60-year history described by Saïdi (1987);

- North Sea chalky reservoirs, including the Ekofisk complex (with oil reserves in Danian and Maastrichtian chalks), are another type of fractured reservoirs of type (b) or (c) with a very high contribution of the matrix to the oil reserves and also a variable contribution to the reservoir permeability;

- dolomitized and karstic or vuggy limestone reservoirs, generally involving a lesser importance of the matrix in the oil reserves (type (b) to type (a)), are found in a good number of provinces, among which some Mexican carbonate provinces such as the Campeche gulf with the giant Cantarell complex, and a good number of North American 
provinces, such as the Rocky Mountains foothills in Western Canada, where fractures enhance the productivity of tight low-porosity dolomitized carbonate formations of Paleozoic age (Mississippian/Devonian carbonates). Karstic reservoirs may not be considered as typical fractured reservoirs but more as the result of an intense and multi-stage diagenesis of some carbonate reservoirs consisting in weathering, dissolution and re-crystallization phenomena. The latter are caused by exogene chemicallyaggressive solutions that circulate along preferential flow paths that may be of sedimentary origin like highly-permeable strata or inter-strata, or of structural origin like fractures. The process results in a complex 3D network of conduits associated with cavities, vugs of various size along those flow paths, whereas the contribution of the matrix, even if it remains porous, tends to become negligible due to the extreme permeability contrast generated by that specific diagenesis;

- old sandstone reservoirs, of Paleozoic age, are often fractured and in majority of type (a). The following examples can be quoted: the giant Amal field in Libya, the Clair field in UK North Sea, many tight Cambrian to Devonian quartzite reservoirs in Algeria, the giant Romachkino field producing from Devonian sandstones in the Ural-Volga province of Russia;

- to end with this non-exhaustive list of examples, a few fractured fields are even found in plutonic formations, generally granites, more rarely volcanic rocks (Jatibarang in Indonesia). The most typical one is probably White Tiger, the first Vietnamese oil field in size. One can also mention La Paz field in Venezuela. Actually, the oil reserves of such fields are often shared between sedimentary reservoirs and the underlying altered basement rock. Fractured gas fields are less reported because the high mobility (low viscosity) of gas makes the impact of fractures on reservoir production behaviour less apparent than for fractured oil reservoirs. One can quote a few examples however, like Paleozoic sandstone reservoirs in Algeria, the Rocky Mountains Paleozoic formations already evoked before, several small gas fields (Meillon, etc.) in the South-West of France. Although generally not considered as conventional gas reservoirs, fractured shale gas reservoirs can also be quoted as they hold potentially-high reserves, especially in North America.

\section{Fractured Reservoirs Significance}

Most major oil \& gas-producing countries are concerned with the presence of fractures in their reservoirs. Indeed, in addition to the few typical examples given above, many other fractured reservoirs are found in other hydrocarbon provinces and other countries. The presence of fractures and/or conductive faults is either acknowledged or suspected in many carbonate fields of the Middle East platform (in Saudi Arabia, the United Arab Emirates, Qatar and Oman), producing from the Permian (Khuff), Upper Jurassic (Arab zone), Cretaceous (Thamama, etc.), Eocene (Damman) formations. China, Central and South Asian countries, South American countries along the Andean Belt, European countries (Italy, etc.) also produce a substantial amount of oil from fractured reservoirs.

Considering the share of carbonate reservoirs in world oil reserves plus the hydrocarbon reserves found in the generally-siliceous reservoirs of Paleozoic age, one can reasonably assume that around half of oil reserves are found in reservoirs where the question of a possible fracture impact on productivity and recovery is worth being addressed, although such an impact will turn out to be effective or sensitive for only a fraction of them, depending on such important properties as fracture density, connectivity and conductivity. This coarse figure stems from the shares of carbonate reservoirs, $40 \%$, and of primary age reservoirs, $13 \%$, in the world oil reserves estimated by Perrodon (1980) on the basis of giant oil fields defined as fields with oil reserves of more than 500 million barrels (around 70 million tons).

Beyond the question of considering a reservoir as fractured or not, the main concern is assessing to which extent the presence of fractures influences field reserves. The concern about fault/fracture impact on production and recovery has grown during the last decade and efforts have been made to forecast potentially-related problems of production. One heuristic reason was the occurrence of unexpected production behaviour, such as early water breakthroughs, in some fields initially considered as non-fractured. Other technical reasons include the improved seismic resolution of reservoir structure and discontinuities, the availability of sophisticated fracture/fault detection tools, and the recent capabilities to analyze those pieces of information and integrate them for field flow modelling purposes. Nowadays, provided a minimum amount of data, the detection of fractures and the evaluation of their possible flow impact are carried out earlier in field life to optimize the placement of wells and the recovery method accordingly: fracture impact assessment has been carried out as part of most reservoir engineering studies.

A recent published survey from Allan and Sun (2003) indicates that the ultimate recovery factor for 56 fractured oil reservoirs with reliable data ranges from less than $10 \%$ to $60-70 \%$, an estimated range already reported by Firoozabadi (2000). Analyzing recovery data into more detail, the authors conclude that such scattered values are not only the result of reservoir characteristics, i.e. the fracture network, the aquifer drive and the matrix properties, but also the consequence of adequate or inadequate recovery methods and production management. That is, more than for other types of reservoirs, reserves maximization calls for joint efforts of geoscientists and reservoir engineers, 
i.e. for an integrated static/dynamic assessment of fractures. Three aspects contribute to that assessment:

- a detection and diagnosis of the flow-impacting fractures thanks to meaningful pieces of information;

- the transcription, without any loss, of that information into a reservoir model designed for flow simulation;

- the capability of that model to actually simulate reservoirrepresentative recovery processes.

Hereafter, the first two aspects are discussed as a brief introduction to the ultimate flow simulation step that is largely developed in two dedicated articles of the present journal issue.

\section{USE OF ENGINEERING DATA TO DIAGNOSE FRACTURE IMPACT ON FLOW}

A review is made of field flow information that may correspond to the "dynamic" expression of fractures. Such indications are not sufficient alone to conclude in favour of the presence of fractures but needs to be confronted to geological information. Apart from image logs and production profiles that give a direct evidence of the presence of fractures, the use of other logs that may be sensitive to fracture presence and properties will not be evoked because their interpretation is most always case-specific and requires confrontation with other logs or pieces of information.

\subsection{Engineering Data Review}

Various basic engineering data are influenced by the presence of fractures. Starting from initial to advanced stages of field development, they include drilling information, productivity/ injectivity and well test data confronted to matrix-representative core permeabilities, production logging, and later on the field dynamic history. Regarding that field history, unexpected events or a peculiar evolution of production have to be thoroughly analyzed in order to confirm that they actually result from the presence of fractures/faults, rather than from other flow heterogeneity sources such as sedimentary and petrophysical contrasts for instance. The reader's attention is drawn again on the necessity to consider the various origins of dynamic responses before concluding on the presence of fractures or faults.

\subsubsection{Drilling Information}

Mud loss data can now be recorded with sufficient accuracy and sudden losses may be due to the presence of a conductive fracture at the considered well depth (Dyke et al., 1995; Verga et al., 2000). These authors determined the hydraulic width of a fracture by solving, with a proper fluid rheology, the flow equation within the fracture modelled as the space between parallel walls. Following this approach, they found that only fairly-wide fractures, with an hydraulic width exceeding around $0.2 \mathrm{~mm}$, could be detected.

On a qualitative point of view, an increased rate of penetration may indicate the presence of fractured levels, with generally a poor core sample recovery for cored wells.

\subsubsection{Well Flow Behaviour}

\section{Well Testing}

Transient flow tests enable to identify and characterise flowing features. Regarding fractured/faulted reservoirs, transient tests may give information on the fluid capacity contrast and the exchange factor between the fracture and matrix media of a diffusely-fractured porous reservoir, namely of a "dualporosity" reservoir. They may also indicate the presence of a fault and its distance from wellbore.

Whereas theoretical models of fractured reservoirs predict typical responses, well test data do not often show such a behaviour for several reasons such as wellbore storage effects (unless well is shut in downhole), or an insufficient fracture network connectivity with the wellbore especially in the case of vertical wells. On the opposite, fields with high permeability contrasts between layers can give well test responses analogous to dual-media responses. At the extreme limit, very thin super-permeable streaks can hardly be dissociated from fractures due to their low capacity and high permeability.

\section{Well Productivity}

The overall productivity of a tested well is already a valuable information if confronted to other well information, namely, facies and fracture distribution along the wellbore, petrophysical data measured on core samples, and other flow data such as production logging (PLT, flowmeter). A matrix contribution to the overall formation permeability that is several times less than the fracture contribution leads to suspect the presence of conductive fractures or of geological objects equivalent to fractures regarding their impact on flow. Of course, these matrix and fracture contributions to reservoir deliverability have to be evaluated on the same reservoir section. Regarding the evaluation of matrix contribution, the thickness-weighted core permeabilities have to be representative of the reservoir section under consideration, which may be problematic in case of missing cores or partial completion.

The limited number of interpreted well tests is another reason for analysing Productivity Indices (PI) to assess the possible impact of fractures on production in the different field regions. However, because PIs do not only reflect reservoir permeability but also near-wellbore effects (negative or positive skin), prior analysis of the latter effects on interpreted well tests is recommended. As a preliminary/tentative field diagnosis of fracture impact on flows, the normalised PI values (PI divided by completion length) of all the wells can then be correlated to the fracture density along the completed wellbore section or other possible fracture indicators such as the distance to the nearest fault. 


\section{Production Logging}

Production logging of the whole completed section of the well enables to specify the contribution of each facies to well test permeability, which can be compared to core-derived facies permeabilities in order to derive the contribution of fractures to reservoir permeability facies by facies. These contributions can then be confronted to the fracture densities measured on cores or image logs of the respective facies, in order to qualify the conductivity of fracture sets defined by the geologist. An example of such a comparison is shown in Figure 1 for a fractured sandstone reservoir (Guaiquirian et al., 2007). This valuable quantification of fracture flow impact assumes a sufficient sensitivity of the tool response, that is contrasted and measurable rates, which discards heavy/viscous oil reservoirs and/or low-conductivity fracture sets.

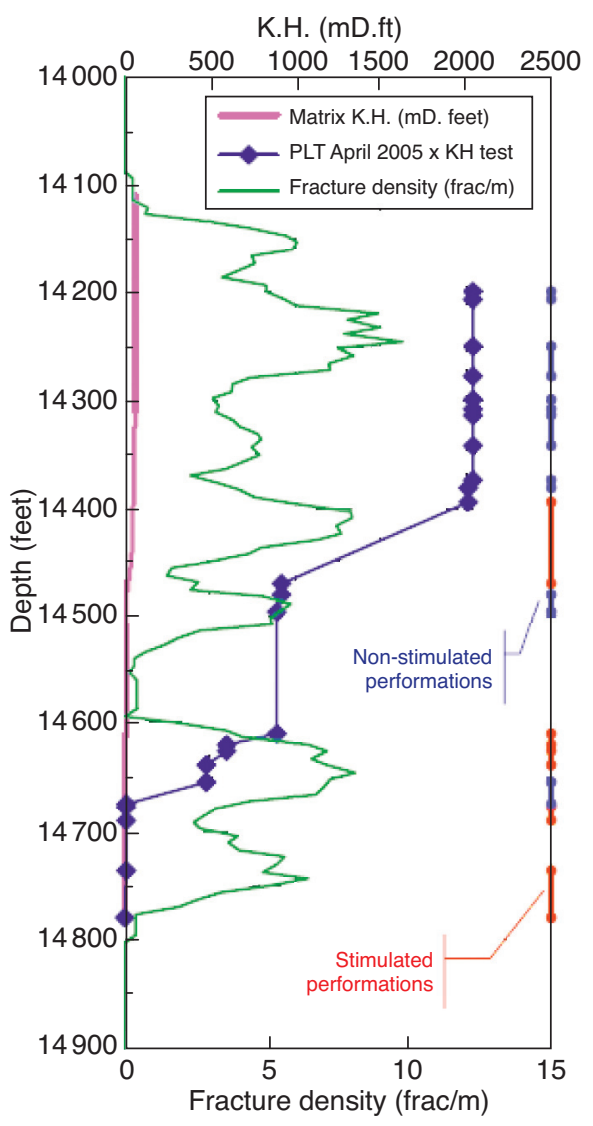

Figure 1

Composite well log confronting:

- the fracture density profile derived from borehole image (green);

- the cumulative matrix permeability-thickness $(\mathrm{KH})$ product (pink);

- the cumulative (fracture + matrix) $\mathrm{KH}$ profile derived from a flow-meter log and calibrated to the well test KH.

(After Guaiquirian et al., 2007).
Note that fracture contribution to well production can be correlated with structural, fault and/or facies maps, for subsequent fracture modelling purposes.

\subsubsection{Field Production History}

Production history is of course an indicator of the effective role played by fractures during production, although the reservoir engineer generally wishes to anticipate such effects. Most of the following information may be meaningful in terms of fracture flow impact: distribution maps of well productivity/injectivity and cumulated production, pressure maps, fluid contact evolution with time, breakthrough times, trend in the watercut and GOR evolution with time. The analysis of such reservoir engineering data is becoming common practice for fields with sparse fracture information (especially borehole image logs and flowmeters) from a few wells, but a more extensive set of long-term production data. Two recent examples concern Middle East fields (Cosentino et al., 2002; Ozkaya and Richard, 2006) where water injection resulted in earlier water breakthrough than expected (Fig. 2). Subsequent matrix and fracture/fault characterization was then undertaken from seismic, wellbore and production data and made possible to identify fracture swarms or corridors as the conductive features responsible for early water breakthrough.

For already-developed fields, multiphase production analysis is indeed essential for further production optimization or re-development. The single-phase production time elapsed before breakthrough at each well has to be correlated to wellbore fracture data (if available) or to structural data (distance to nearest fault, position in the reservoir structure, etc.). Multiphase production data, such as for instance the rate of water-oil ratio increase, can also give information regarding the contribution of matrix-fracture transfers or cross-flows from low-permeability layers to high-permeability streaks if the fracture origin of water arrival cannot be ascertained.

For multi-compartment fields, the evolution during production of pressures and fluid composition (oil PVT properties, water salinity) is also essential to identify possible communications between reservoir compartments in connection with the presence of conductive faults.

\section{2 "Dynamic Fracture" Analysis in Practice}

Assessing the presence of fractures and their role on flow requires integrating and cross-checking various types of information. A methodological framework is proposed below, keeping in mind that it has to be adapted to the amount, type and quality of available data:

- collection and diagnosis of fracture indicators on a wellby-well basis. For each well of a given type (horizontal vs vertical, production vs injection, completion type, etc.), all 


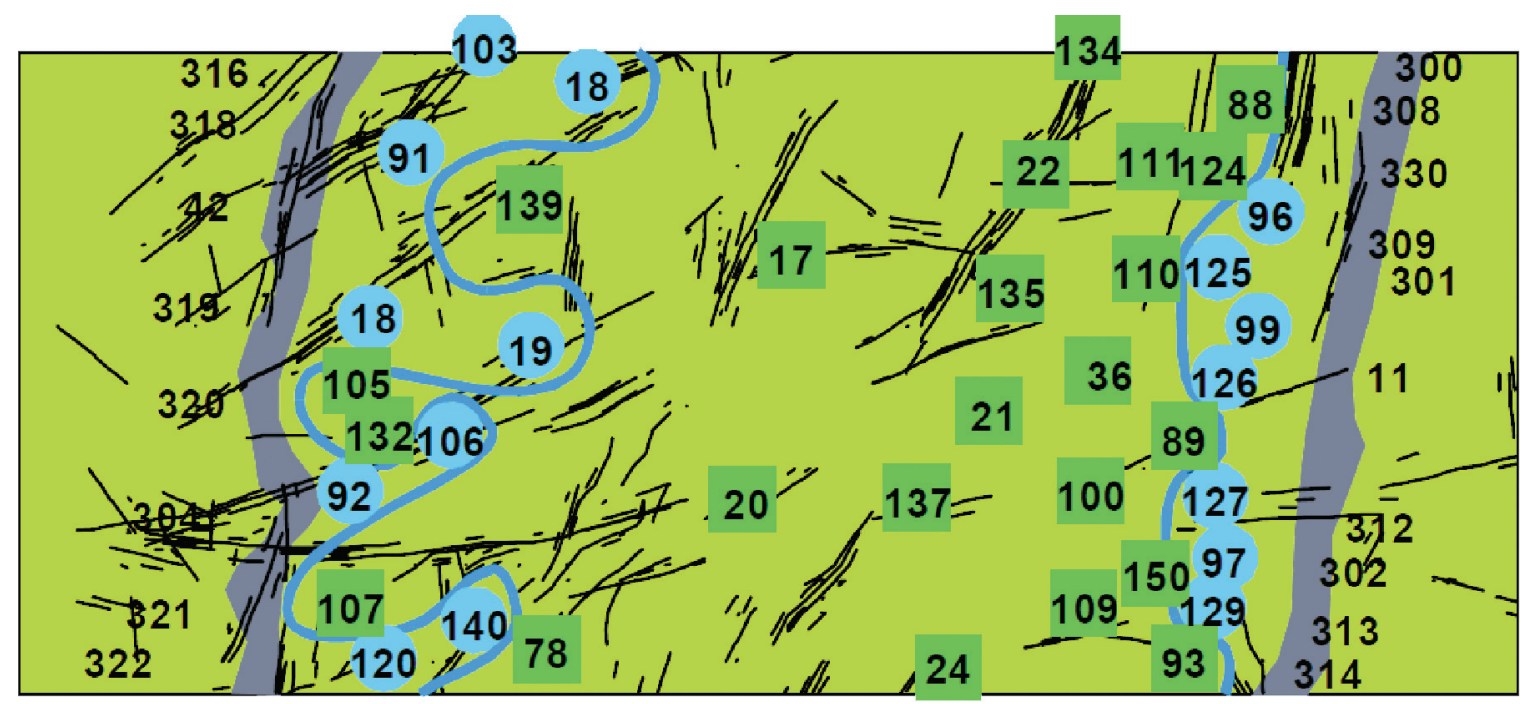

Figure 2

Irregular water front progression in a giant field crossed by sub-seismic faults: the original position of the water-oil contact is shown as grey bands and the present position as blue lines; wet wells are shown as blue circles and dry wells as green squares (After Cosentino et al., 2002).

available indicators among the following are collected, analysed and normalized for comparison purposes:

- well-test to core-derived permeability ratio,

- mud losses report,

- flow rate peaks on production log,

- transient well test flow behaviour,

- productivity index normalised to completion length (in the absence of interpreted well test data),

- time elapsed before water breakthrough,

- cumulative oil production, etc.;

- classification of wells according to the quantitative or qualitative values of previously-defined fracture indicators;

- mapping the distribution of wells according to fracture indicator values: evidence of field regions or correlations between wells;

- interpreting the distribution of fracture indicators values over the field through the analysis of other production information such as:

- the displacement of fluid contacts with time,

- the distribution map of produced fluids composition (water salinity if injected and connate/aquifer water compositions differ),

- pressure and material balance analysis in the presence of multiple reservoirs, etc.;

and through the integration of geological information such as:

- fracture set orientations,

- regional stress orientation;
- selecting a conceptual flow model of the field that integrates the flow-impacting fractures that have been identified before, while taking into account their necessary geological interpretation as recalled at the end of this section;

- further quantifying the conductivities of faults or fracture sets intercepting wells where production profiles have been measured along with a core-derived matrix permeability profile.

To conclude, many reservoir engineering dynamic data may be related to the presence of conductive fractures. Obviously, these sources are not often available all together for a given reservoir. In addition, each reservoir case calls for specific flow diagnosis, for instance the discrimination between "useful" and non-conductive fracture sets from production logging (Fonta et al., 2007). The methodology definitely has to be defined as a function of available fracturerelated data and of the objective assigned to the study.

Organising, cross-checking and correlating those data is essential to gain a reliable understanding of fracture impact on field production and provide a sound basis for subsequent field flow modelling. However, their correlation with other "static" information about fractures and faults provided by the geologist remains essential. Actually, the reservoir engineer has to work in close and permanent collaboration with the geologist:

- to properly interpret the origin of preferential flowing features which may be of sedimentary origin in addition to or instead of structural origin, i.e. fracture-related; 
- to understand the mechanical genesis of fracture sets in order to predict their distribution within the reservoir;

- to identify the origin of determinant fracture properties such as their conductivity, in order to assess the probable fracture flow impact in reservoir zones already characterized from seismic and geological information but not yet or sparsely drilled and produced;

- to properly design acquisition campaigns of further dynamic data.

If data/information cross-checking concludes in favour of the presence of fractures and of their effective role on flow, then we have to integrate them into the reservoir flow model. However, that integration cannot be straightforward because the fracture network is complex, constituted of fracture sets with different orientations, densities, lengths, conductivities, etc. To deal with that complexity, a conceptual flow model has to be selected and parameterized in order that reservoir production scenarios can be simulated within reasonable computation times and with a satisfactory reliability.

\section{SETTING UP A RESERVOIR FLOW MODEL}

\subsection{Selecting a Conceptual Flow Model}

\subsubsection{A Challenging lissue}

Setting up the flow model of a naturally-fractured reservoir remains a challenging issue for the reservoir engineer because he has to find a compromise to meet with the following requirements:

- integrate the geological information regarding the geometry and distribution of faults and fractures: the link between the geological fracture model and the flow model has to be maintained for the interpretation of the role of fractures on production and for subsequent up-dating;

- reproduce and/or predict the dynamic field response for the production scenario(s) under consideration: indeed, the impact of fracture parameters is closely depending on the involved recovery mechanism(s);

- take into account limited computation capabilities of industrial simulation software.

Most often, the 3D geological model of faults and fractures is too complex to be used as a direct input for a reservoir simulation software. In fact, typical horizontal and vertical dimensions of a reservoir simulator cell are respectively in the order of the hectometre and of the meter-decametre due to limitations in the size of field-scale numerical models. Within such cells, the centimetric to decametric fractures are assumed to behave like an equivalent medium (Warren and Root, 1963), that is, the flow properties of the small-scale fracture network are assumed representative at cell scale and describable by a permeability tensor. On the opposite side, hectometric to kilometric faults crossing the reservoir cannot be incorporated into the equivalent fracture medium defined at cell scale but have to be discretized individually. The intermediate scale of sub-seismic faults and/or fracture swarms poses a specific problem as they are too numerous to be modelled explicitly and too large to behave as an equivalent medium at simulator cell scale. In addition to their scale, the flow properties of fractures/faults and their interactions with the surrounding medium are determining criteria for the choice of a representative and predictive flow model. Methodological criteria are proposed hereafter, keeping in mind that they have to be tuned to the practical field situation under consideration and to the objectives of the reservoir simulation study. For instance, the effort to be devoted to a consistent integration of fracture information into the field model differs considerably whether one has to simulate a short depletion history of an under-saturated oil reservoir or the re-development of an already-waterflooded or -gasflooded mature field.

Most generally, three criteria drive the choice of a conceptual flow model:

- the fracture scale compared to that of model grid cells, that determines the existence of an equivalent fracture medium at the grid cell scale;

- the connectivity of the fracture network and the continuity of the matrix medium, that determine the flow continuity between cells of a given medium;

- the time scale of flow interaction (or transfer) between media compared to the time scale of fluid transport within a given medium.

That is, a hierarchical approach with respect to scale is necessary but not sufficient. In particular, matrix medium properties - permeability, etc. - are also involved as they determine the kinetics of interaction between matrix and fractures.

\subsubsection{Our Methodology}

Let us explicit our methodology of choice (Fig. 3, after Bourbiaux et al., 2002). We have to deal with two main media, namely matrix and fractures of different characteristic scales. The porous medium entities delimited by fractures are denoted as "matrix blocks" and the characteristic dimensions of these matrix blocks are denoted as the "block size":

- the scales, denoted as $l$, can be classified with respect to the model grid cell dimensions, denoted as $\Delta x$;

- the connectivity/continuity of fracture objects and of the matrix medium also has to be taken into account at various scales;

- the kinetics of matrix-fracture interactions has to be assessed in comparison with the fracture medium flow. Let us assume $\Delta t$ as the typical simulation timestep required to describe with a sufficient accuracy the flow progress in the fracture medium alone. That characteristic time for 
flow in fractures may be of the order of one week or month for a field produced over one or few decades. Then, a quasi-static equilibrium of pressure, saturation, composition or temperature between matrix and fracture media can be reasonably assumed if this equilibrium is established within a time, $t_{e}$, that is much less than $\Delta t$. The characteristic time for matrix-fracture transfers, $t_{e}$, can be estimated from analytical solutions, but for simple transfer mechanisms, diffusive for instance, under given initial and boundary conditions of the matrix blocks. Otherwise, numerical solutions can be computed on the fine-grid model of a matrix block limited by fractures.

1 st case: fracture scale (average length), $l_{f}$, is less than cell size, $\Delta x$.

(1a) Fractures are disconnected: this situation occurs in the presence of microfractures inducing a permeability anisotropy but no dual-medium flow since the matrix medium is the single medium ensuring the flow continuity from one cell to another. A single-medium model has to be constructed then, with equivalent matrix flow properties that incorporate the local contribution of microfractures to cell-scale flow.

(1b) Fractures constitute a connected network and $t_{e}$ is less than $\Delta t$ : this situation concerns densely-fractured media delimiting small matrix blocks that exchange fluids very rapidly with the fracture network when solicited by the latter. This quasi-static equilibrium between matrix and fractures is very often satisfied in single-phase flow conditions but not so often in multi-phase conditions. As before, a single-medium model can be used with equivalent permeabilities accounting for the contribution of both media.

(1c) Fractures constitute a connected network and $t_{e}$ is higher than $\Delta t$ : matrix-fracture transfers are delayed with respect to the fluids transfer taking place in the fracture network. In that case, the use of a dual-medium model is recommended to reproduce the kinetics of matrix-fracture transfer:

- if the fractures delimit blocks that have little or no flow interaction together, then the matrix medium only acts as a local source of fluids for the fracture medium and a dualporosity single-permeability approach is well-suited;

- but if flow interactions are taking place between successive matrix blocks, either through the existence of porous/ non-porous bridges between blocks ("physical" or "static continuity") or via re-imbibition phenomena ("dynamic continuity"), then both the fracture medium and the matrix medium constitute continua for flows between cells: this situation calls for the adoption of a dual-porosity dualpermeability model. Such a "dynamic continuity" often has to be taken into account for the simulation of gravitydriven production processes.

Although the existence of flow interactions between matrix blocks has been demonstrated and quantified from laboratory experiments, a priori assessment of such interactions is much more speculative when dealing with an underground reservoir. The geological history of fractures, including their diagenetic phenomena, may be informative but in practice, it is mainly through a sensitivity study of the reservoir history match to the assumption of matrix blocks interaction that the choice between a single- or a dual-permeability model will be made.

2nd case: fractures have an average dimension, $l_{f}$, close to or exceeding cell size, $\Delta x$, but remain far below reservoir scale and less than the well spacing.

$(2 \mathrm{a}, 2 \mathrm{~b})$ Fractures form a connected network: this situation remains similar to previous situations $1 \mathrm{~b}$ and $1 \mathrm{c}$ depending on the rapidity of matrix-fracture transfers (quasi-static equilibrium for case $2 \mathrm{a}$ equivalent to case $1 \mathrm{~b}$, and slow transfers for case $2 \mathrm{~b}$ equivalent to case $1 \mathrm{c})$. In multiphase flow situations, $t_{e}$ is generally higher than $\Delta t$, hence the adoption of a dual-medium model is often required. In addition, since the dimensions of blocks are of the same order of magnitude or exceed cell size, matrix flows from cell to cell have to be taken into account. That is, situation $2 \mathrm{~b}$ often calls for the choice of a dual-porosity dual-permeability model, or at least much more often than in previous case 1c.

(2c) Fractures do not constitute a connected network all over the reservoir, some areas being sometimes not fractured at all: this situation is analogous to previous case $2 \mathrm{a}$ in the fractured reservoir regions. Therefore, the dual-medium modelling approach remains adapted. However, if a singlepermeability model is selected, the latter should be implemented within a simulator that offers the possibility to switch to a matrix single-medium model in non-fractured areas. The dual-porosity dual-permeability approach intrinsically offers such a possibility, as both matrix and fracture media are then considered as two continua.

Case 2c may correspond to sparsely-distributed sub-seismic faults or fracture swarms with a pluri-hectometric horizontal scale and a high vertical extension. Because of their low to nil throw, they may be undetectable individually from seismic surveys. However, due to their size and their nonuniform distribution, they may closely influence the reservoir-scale flow behaviour, and be the origin of early injected fluid breakthroughs for instance. An accurate simulation of fluid flows along these large fractures with a dual-medium model is required, taking into account in particular the orientation and connectivity of those objects within and between cells through discrete fracture cell transmissivities (Cosentino et al., 2002). For the above reasons, sub-seismic faults or fracture swarms remain a weak point and a real source of uncertainties for fractured reservoir flow model. This justifies collaborative efforts of geologists and reservoir engineers to better characterize this type of fractures and to capture their impact on the production history. 


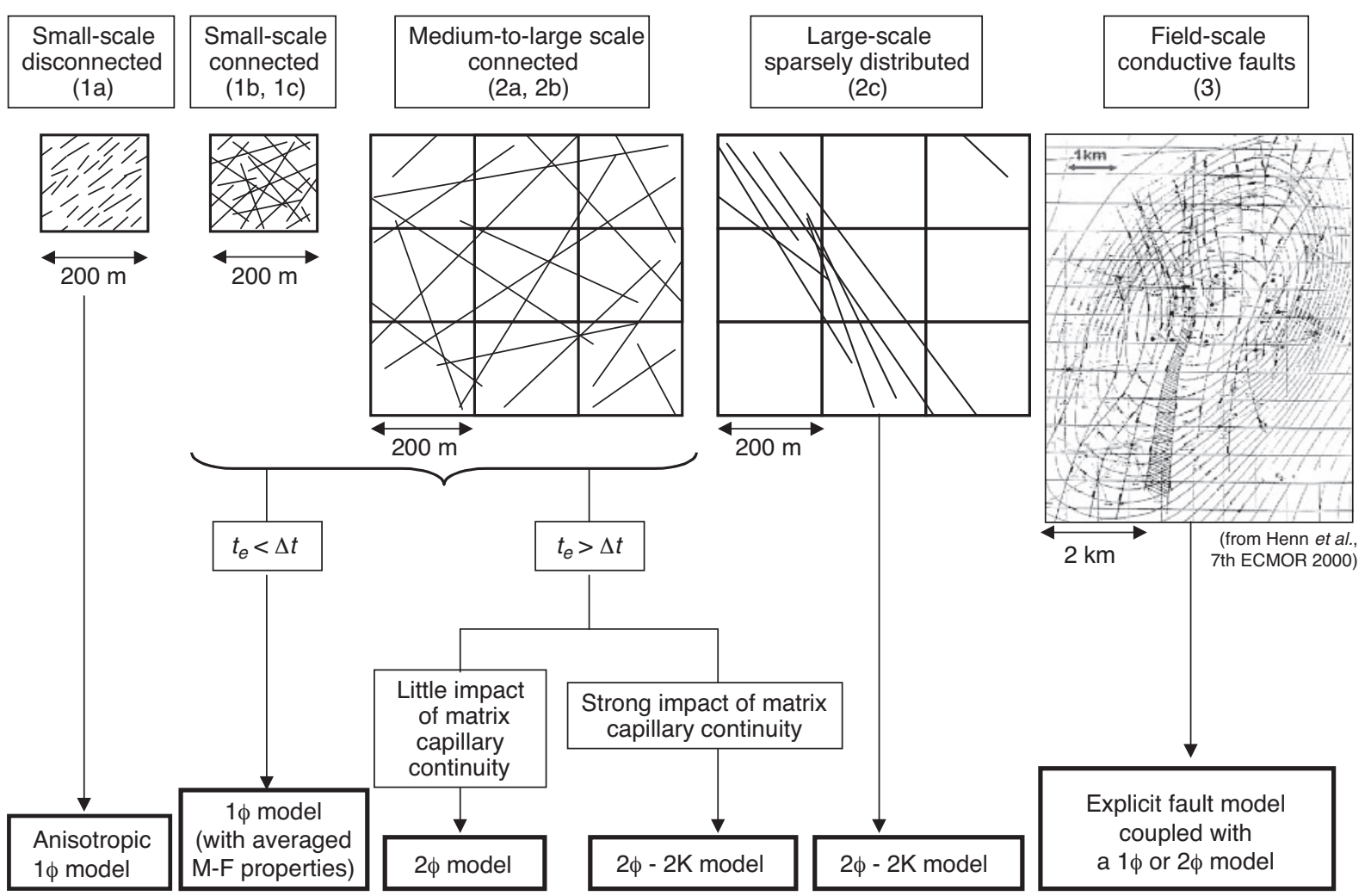

Figure 3

Guiding lines for selecting the flow modelling approach of a fractured reservoir (After Bourbiaux et al., 2002).

3rd case: fractures have a dimension, $l_{f}$, largely exceeding cell size, $\Delta x$, and constitute major identified conduits, which vertically cross-cut the reservoir with possible throw between delimited faults.

This situation is encountered in some carbonate fields where conductive faults contribute to fluid transport at reservoir scale and constitute bypass between wells. An explicit representation of those faults is necessary to properly match the multiphase field flow behaviour, that often involves early breakthroughs at given well locations. As a discrete representation of each individual fault is computer-intensive, a specific "conductive fault model" (Henn et al., 2004) may be adopted. The segregated flow concept that underlies that model avoids any vertical gridding of conductive faults and the related numerical constraints.

The various situations described above assume the presence of fractures/faults crossing a porous matrix. Two particular situations are worth being mentioned:

- non-porous fractured reservoirs: a conventional singlemedium model is generally well suited; however, if major fractures control the large-scale flow of fluids produced from microfractures and/or vugs having no direct access to macro-fractures, then a dual-porosity approach may still be required;
- conversely, non-fractured heterogeneous reservoirs including numerous high-permeability beds sparsely distributed among tighter layers: such reservoirs behave like porous fractured reservoirs and can be satisfactorily represented by a dual-porosity dual-permeability model.

\subsection{Assigning Properties to the Flow Model}

Once a modelling approach is chosen, effective or equivalent flow properties have to be assigned to each cell of the simulation model. These properties refer to the continua assumed representative of the actual fractured medium at the flow model resolution scale, i.e. at the cell (or grid-block) scale.

The determination of such effective, or up-scaled, properties has long remained a fractured reservoir modelling obstacle. The recent possibility to compute flows on discrete models of geological fracture networks (Bourbiaux et al., 1998; Sarda et al., 2002; Matthaï et al., 2007 among other authors) was a real breakthrough that contributed to the effective integration of fracture geology into fractured reservoir engineering (Sabathier et al., 1998). 
In the following, the determination of the following equivalent parameters is discussed:

- for single-porosity models: equivalent permeabilities for both single-phase and multiphase conditions;

- for dual-porosity models: equivalent single-phase fracture permeabilities and an equivalent matrix-fracture transfer parameter that may consist in the dimensions of an equivalent block or in a shape factor.

Note that for dual-porosity models, the necessity to determine multiphase effective (up-scaled) parameters is avoided if the two following conditions are actually satisfied together:

- the multiphase matrix-fracture exchanges taking place at the cell scale can be formulated through the use of a single exchange parameter (for instance, a single matrix block of given size and shape);

- the formulas used to compute the matrix-fracture transfer fluxes for a cell represent the involved transfer mechanisms and are sufficiently accurate.

The predictability improvement of upscaled formulas for multiphase matrix-fracture transfers has long been a matter of research and remains a subject of new developments to better capture the transient character of exchanges (Bourbiaux et al., 1999; Sarma and Aziz, 2006; Lu et al., 2008; van Heel et al., 2008, among numerous authors). Special attention is dedicated to this question in the two other articles of this journal issue that are dedicated to the state of the art in fractured reservoir simulation (Lemonnier and Bourbiaux, 2010).

\subsubsection{Effective Properties of a Single-Porosity Model}

The effective properties of a single-porosity model, in particular the effective permeabilities, have to represent both contributions of matrix and fracture media to flow and transport, as well as matrix-fracture exchanges. However, the approximation of effective permeabilities as the sum of the permeabilities of each separate medium is valid only when the two media behave as two superposed continua and matrix-fracture transfers are very rapid, i.e. when the two media are in quasi-static equilibrium. Such conditions may not be satisfied, especially in the presence of slowly-interacting media and/or in the presence of poorly-connected fractures. In the latter situation, the determination of the effective permeability of both media requires to solve coupled equations describing flow in the discrete model of the fracture network and the matrix medium, as proposed for instance by Lough et al. (1997). However, numerical computation of coupled matrix and fracture flows on discrete models is not yet conceivable for field-scale models involving hundreds of thousands to millions cells. Moreover, the problem of up-scaling matrix and fracture flow contributions is still more complex under multiphase conditions. Actually, the determination of effective multiphase permeabilities, or of pseudo-relative permeabilities, has no general solution. Except for situations of quasi-instantaneous matrix-fracture transfer times, the solution for pseudo-relative permeabilities is far from unique as it closely depends on the flow mechanism under consideration, the fracture flow conditions in terms of rate, or saturation, composition, temperature, and on the field flow history. Different conventional methods exist to compute those parameters, such as for instance the numerical resolution of steady-state flows on fine-grid models with various fractional flows of the fluids in presence. However, none of them has a general scope of application.

For all reasons stated above, dual-medium (or dual-porosity) models are recommended for most fractured reservoirs studies involving a complex production history.

\subsubsection{Effective Properties of a Dual-Porosity Model}

The basic cell input of a dual-porosity simulation model include the equivalent fracture permeabilities and a matrixfracture exchange parameter. For the sake of consistency, these cell-up-scaled parameters have to be determined from the geological model of fractures for each cell or group of cells. The determination of the (up-scaled) dual-medium flow properties of fractured media has received considerable attention from researchers in both sectors of petroleum and water resources management. A discussion of these approaches can be found in Landereau et al. (2001), along with an application of the volume averaging method.

The first published works generally concerned the determination of equivalent permeabilities.

\section{Equivalent Fracture Permeabilities}

Either analytical or numerical methods can be adopted. Analytical methods as the one proposed by Oda (1985), use the statistical parameters of fracture sets as calculation input, without requiring any Discrete Fracture Network (DFN) modelling and discretization. However, they are valid under certain conditions, essentially a high density and connectivity of the conductive fracture network, which are not always fulfilled in practice. These restrictive conditions motivated the development of numerical methods, as the ones proposed early by Long et al. (1985), Cacas et al. (1990), Odling (1992) among other authors. The flow problem addressed by these authors concerned non-porous fractured media such as granites, and methods differed in the technique of discretization of the Fracture Network. Basically, numerical methods for computing equivalent fracture permeabilities consist in solving a steady-state flow problem on the discrete fracture network with application of Poiseuille's formula for fracture flows, similarly to a resistor network equivalence problem. More recently, a numerical method inherited from the previous ones, but based on a minimum discretization of the DFN, was developed for application to oil and gas fractured reservoir engineering studies (Bourbiaux et al., 1998), i.e. for dual-porosity models involving a large number of cells. For all that, flow computation on large and/or complex discrete fracture networks remains computer-intensive, even with 
ever-increasing computational capabilities. For this reason, a complementary use of analytical and numerical methods looks like an optimal compromise for up-scaling fracture permeabilities for field-scale engineering studies. In this respect, Delorme and Bourbiaux (2008) proposed a methodology of choice between both methods that is based on a connectivity index calculated from the statistical parameters of fracture sets as characterized by the geologist. Garcia et al. (2007) made also use of both methods to take into account the scale dependence of effective fracture permeability, whether it is calculated within one layer or over a stack of layers.

\section{Matrix-Fracture Exchange Parameter}

Depending on the industrial simulator under consideration, the exchange parameter may consist in the dimensions of a characteristic (or "equivalent") matrix block or in a shape factor, the latter being inversely proportional to the square of the characteristic block dimension(s). Considering that fractures are most often sub-orthogonal to bed limits, then the matrix medium presents a certain degree of capillary continuity in the orthogonal direction to bedding planes. Therefore, the equivalent matrix block (or shape factor) determination problem turns out to be essentially two-dimensional. For each layer or group of layers having similar fracturing features, it consists in identifying a square or rectangular equivalent block section from the geological fracture network. To this end, a fast geometrical method (Sarda et al., 1997) was developed to compute a characteristic function of matrixfracture exchanges in both the geological and the equivalent fractured media. That function expresses the $2 \mathrm{D}$ piston-type penetration of a fluid front into the matrix block(s), as a simplified representation of various possible diffusive mechanisms driving matrix-fracture transfer.

Lastly, an equivalent vertical block height may have to be assessed to compute the contribution of gravity forces to matrix-fracture exchanges at the model cell scale. That requirement concerns the dual-porosity single-permeability models of reservoirs where the assumption of an interrupted capillary continuity of matrix blocks is justified by structural or sedimentary reasons reservoirs such as a significant dip, tight intervals or super-permeable strata. Even though a careful geological characterization of reservoir structure and facies is helpful to qualitatively assess the matrix flow continuity assumption, uncertainty on equivalent block height values is inevitable and necessarily calls for a tuning through the history matching of the field production.

\section{FRACTURED RESERVOIR MODELLING: PROGRESS PERSPECTIVES}

The growing awareness of the fracture impact on field production has been the incentive for significant advances in the characterization and modelling of fractured reservoirs in the recent years. Geosciences and reservoir engineering integration resulted in better-constrained flow models of fractured reservoirs, and unveiled at the same time limitations calling for further progress:

- at an intermediate scale between small-scale diffuse fractures and seismic faults, sub-seismic faults and fracture swarms remain a difficult issue, as they can neither be treated as part of a fracture continuum, nor be defined as discrete objects: pending their detection by high-resolution seismic, stochastic models of sub-seismic faults need to be further constrained from other geological information and from dynamic data or production history;

- high-performance methodologies for characterizing and up-scaling the flow properties of fracture networks are needed to parameterize full-field flow models inherited from ever more complex geological fracture models;

- multi-scale expression of natural fracturing calls for flow models that couple flow continua and discrete objects.

Once a field-representative flow model has been set up and parameterized, the reliability of a fractured reservoir flow simulation still depends on the adequate transcription of the physical flow mechanisms taking place in and between the constitutive media, matrix, fractures and/or faults, especially for porous fractured/faulted reservoirs where a dominant share of the field oil reserves are held in the matrix medium. In this respect, two articles of that journal issue (Lemonnier and Bourbiaux, 2010) are dedicated to fractured reservoir simulation with a specific attention to these matrixfracture/fault interactions.

\section{REFERENCES}

Allan J., Qing Sun S. (2003) Controls on Recovery Factor in Fractured Reservoirs: Lessons Learned from 100 Fractured Fields, SPE paper 84590 presented at the SPE Annual Technical Conference and Exhibition, Denver, Co.

Bourbiaux B., Cacas M.C., Sarda S., Sabathier J.C. (1998) A Rapid and Efficient Methodology to Convert Fractured Reservoir Images into a Dual-Porosity Model, Oil Gas Sci. Technol. - Rev. IFP 53, 6, 784-799.

Bourbiaux B., Granet S., Landereau P., Noetinger B., Sarda S., Sabathier J.C. (1999) Scaling Up Matrix-Fracture Transfers in DualPorosity Models: Theory and Application, SPE Paper 56557 presented at the SPE Annual Technical Conference and Exhibition, Houston, TX, 3-6 Oct.

Bourbiaux B., Basquet R., Cacas M.C., Daniel J.M., Sarda S. (2002) An Integrated Workflow to Account for Multi-Scale Fractures in Reservoir Simulation Models: Implementation and Benefits, SPE Paper 78489 presented at the 10th Abu Dhabi International Petroleum Conference and Exhibition (ADIPEC), Abu Dhabi, 13-16 Oct.

Cacas M.C. et al. (1990) Modeling Fracture Flow With a Stochastic Discrete Fracture Network: Calibration and Validation, Water Resour. Res. 26, 3, 479-500.

Cosentino L., Coury Y., Daniel J.M., Manceau E., Ravenne C., van Lingen P., Cole J., Sengul M. (2002) Integrated Study of a Fractured Middle East Reservoir with Stratiform Super-K Intervals Part 2: Upscaling and Dual Media Simulation, SPE Reserv. Eval. Eng. (SPE 76642) 5, 1, 24-32. 
Delorme M., Atfeh B., Allken V., Bourbiaux B. (2008) Upscaling Improvement for Heterogeneous Fractured Reservoir Using a Geostatistical Connectivity Index, Geostats 2008, Santiago, Chile.

Dyke C.G., Wu B., Milton-Tyler D. (1995) Advances in Characterizing Natural-Fracture Permeability from Mud-Log Data, SPE Formation Eval. (SPE 25022), 10, 3, 160-166.

Firoozabadi A. (2000) Recovery Mechanisms in Fractured Reservoirs and Field Performance, J. Can. Petrol. Technol. 39, 11, 13-17.

Fonta O., Al-Ajmi H., Verma N.K., Matar S., Divry V., Al-Qallaf H. (2007) The Fracture Characterization and Fracture Modeling of a Tight Carbonate Reservoir - The Najmah-Sargelu of West Kuwait, SPE Reserv. Eval. Eng. 10, 6, 695-710.

Garcia M, Gouth F., Gosselin O. (2007) Fast and Efficient Modeling and Conditioning of Naturally Fractured Reservoir Models Using Static and Dynamic Data, SPE Paper 107525 presented at the SPE Europec/EAGE Annual Conference and Exhibition, London, UK, 11-14 June.

Guaiquirian L., Gonzalez P., Gonzalez A., Manuela Hernandez M., Le Maux T., Mattioni L., Rouvroy P. (2007) Use of Discrete Fracture Network 'DFN' to Characterize and Model a Naturally Fractured Sandstone Reservoir: Orocual Field, San Juan Formation, Venezuela - A Case Study, SPE Paper 108052 presented at the SPE Latin American and Caribbean Petroleum Engineering Conference, Buenos Aires, Argentina, 15-18 April.

van Heel A.P.G., Boerrigter P.M., van Dorp J.J. (2008) Thermal and Hydraulic Matrix-Fracture Interaction in Dual-Permeability Simulation, SPE Reserv. Eval. Eng. 11, 4, 735-749.

Henn N., Quintard M., Bourbiaux B., Sakthikumar S. (2004) Modelling of Conductive Faults With a Multiscale Approach, Oil Gas Sci. Technol.-Rev. IFP 59, 2, 197-214.

Landereau P., Noetinger B., Quintard M. (2001) Quasi-Steady TwoEquation Models for Diffusive Transport in Fractured Porous Media: Large-Scale Properties for Densely Fractured Systems, $A d v$. Water Resour. 24, 8, 863-876.

Lemonnier P., Bourbiaux B. (2010) Simulation of Naturally Fractured Reservoirs. State of the Art: Part 1 - Physical Mechanisms and Simulator Formulation, Oil Gas Sci. Technol. Rev. IFP (to be published).

Lemonnier P., Bourbiaux B. (2010) Simulation of Naturally Fractured Reservoirs. State of the Art: Part 2 - Matrix-Fracture Transfers and Typical Features of Numerical Studies, Oil Gas Sci. Technol. - Rev. IFP (to be published).

Long J.C.S., Gilmour P., Witherspoon P.A. (1985) A Model for Steady Fluid Flow in Random Three-Dimensional Networks of Disc-Shaped Fractures, Water Resour. Res. 21, 8, 1105-1115.

Lough M.F., Lee S.H., Kamath J. (1997) A New Method to Calculate Effective Permeability of Gridblocks Used in the Simulation of Naturally Fractured Reservoirs, SPE Reserv. Eng. 12, $3,219-224$
Lu H., Di Donato G., Blunt M.J. (2008) General Transfer Functions for Multiphase Flow in Fractured Reservoirs, SPE J. 13, 3, 289-297.

Matthäi S.K., Mezentsev A., Belayneh M. (2007) Finite ElementNode-Centered Finite-Volume Two-Phase Flow Experiments with Fractured Rock Represented by Unstructured Hybrid-Element Meshes, SPE Reserv. Eval. Eng. 10, 6, 740-756.

Nelson R.A. (1985) Geological analysis of naturally fractured reservoirs, Chilingar G.V. (Ed.), Contributions in Petroleum Geology \& Engineering, 1, Gulf Publishing Company.

Oda M. (1985) Permeability Tensor for Discontinuous Rock Masses, Géotechnique 35, 4, 483-495.

Odling N.E. (1992) Permeability of Natural and Simulated Fracture Patterns, Structural and Tectonic Modelling and its Application to Petroleum Geology, Elsevier, Norwegian Petroleum Society (NPF), NPF Special Publication 1, 365-380.

Ozkaya S.I., Richard P.D. (2006) Fractured Reservoir Characterization Using Dynamic Data in a Carbonate Field, Oman, SPE Reserv. Eval. Eng. 9, 3, 227-238.

Perrodon A. (1980) Géodynamique Pétrolière, Masson \& Cie.

Sabathier J.C., Bourbiaux B., Cacas M.C., Sarda S. (1998) A New Approach of Fractured Reservoirs, SPE Paper 39825 prepared for the SPE International Petroleum Conference and Exhibition, Villahermosa, Mexico, 3-5 March.

Saidi A.M. (1987) Reservoir Engineering of Fractured Reservoirs, TOTAL Edition Presse, Paris.

Sarda S., Bourbiaux B., Cacas M.C., Sabathier J.C. (1997) An Innovative Procedure to Compute Equivalent Block Size in a DualPorosity Model, Paper presented at the 9th European Symposium on Improved Oil Recovery, The Hague, The Netherlands, 20-22 Oct.

Sarda S., Jeannin L., Basquet R., Bourbiaux B. (2002) Hydraulic Characterization of Fractured Reservoirs: Simulation on Discrete Fracture Models, SPE Reserv. Eval.Eng. 5, 2, 154-162.

Sarma P., Aziz K. (2006) New Transfer Functions for Simulation of Naturally Fractured Reservoirs with Dual-Porosity Models, SPE J. $\mathbf{1 1}, 3,328-340$.

Verga F.M., Carugo C., Chelini V., Maglione R., De Bacco G. (2000) Detection and Characterization of Fractures in Naturally Fractured Reservoirs, SPE Paper 63266 presented at the SPE Annual Technical Conference and Exhibition, Dallas, Tx, 1-4 Oct.

Warren J.E., Root P.J. (1963) The Behavior of Naturally Fractured Reservoirs, SPE J. Sept., 245-255.

Final manuscript received in June 2009 Published online in March 2010 